\title{
Autologous cartilage grafts for OA: stable 4 years on
}

Autologous chondrocyte implantation (ACI) with a polymer-based graft could have potential as a treatment for focal degenerative cartilage defects, such as those observed in osteoarthritis. The latest results from an ongoing clinical study of patients with osteoarthritic symptoms of the knee who underwent the procedure show that early improvements are maintained 4 years after graft implantation.

ACI is a well established and effective method of repairing focal, traumatic cartilage defects, but its use is limited by the need for a rim of intact cartilage around the defect. In addition, the periosteal flap or collagen sheet that acts as a reservoir for the transplanted cells can be unstable or can increase the risk of complications, often necessitating corrective surgery. The secondgeneration ACI method used in this study, BioSeed $^{{ }_{-}} \mathrm{C}$ (Biotissue Technologies, Freiburg, Germany), cultivates chondrocytes in a three-dimensional matrix of bioresorbable materials, thus avoiding the use of cover materials such as periosteum or collagen sheets. When anchored transosseously, the fibrinpolymer matrix acts as a scaffold that stabilizes the graft. "This approach allows effective cartilage repair even in defects that lack intact cartilage surrounding the defect," says Christian Kaps, one of the study's investigators.

\section{The benefits were still} evident at 4 years, and included improvements in quality of life and pain 77

The 4-year clinical outcomes of BioSeed $^{\circledR}-$ C performed in 19 patients with preoperative radiographic and clinical signs of osteoarthritis have now been reported. The chondrocytes, taken from healthy cartilage in a non-weight-bearing area of the knee, were transplanted into areas of focal degenerative defects. Clinical effects were observed as soon as 6 months after the procedure, and outcomes were significantly improved from baseline after 1 year. The benefits were still evident at
4 years, and included improvements in quality of life and pain. The procedure failed in two patients, who underwent revision surgery and ultimately received total knee endoprosthesis 4 years after ACI. MRI analysis of those two patients at 1 year showed only partial ( $<50 \%)$ filling of the defect with repair cartilage and articular resurfacing. By contrast, MRI in the other 17 patients demonstrated moderate-tocomplete defect filling at 4 years.

The results of this small, observational case-report study suggest that chondrocyte-based cartilage transplants might have the potential to treat not only small traumatic defects but large focal degenerative defects of cartilage. Kaps remarks that this treatment could "close the gap between initial surgical interventions such as lavage or debridement and total joint replacement".

Sarah Price

Original article Kreuz, P. C. et al. Treatment of foca degenerative cartilage defects with polymer-based autologous chondrocyte grafts: four year clinical results. Arthritis Res. Ther. 11, R33 (2009). 